\title{
Diagnostic Accuracy of Screening Arterial Spin-Labeling MRI Using Hadamard Encoding for the Detection of Reduced CBF in Adult Patients with Ischemic Moyamoya Disease
}

\author{
(D). Setta, (D) T. Matsuda, (D) M. Sasaki, (D). Chiba, (D). Fujiwara, (D). Kobayashi, (D) K. Yoshida, (D). Kubo, (D) M. Suzuki, \\ (D) K. Yoshioka, and (D) K. Ogasawara
}

\begin{abstract}
BACKGROUND AND PURPOSE: Adult patients with ischemic Moyamoya disease are advised to undergo selective revascularization surgery based on cerebral hemodynamics. The purpose of this study was to determine the diagnostic accuracy of arterial spinlabeling MR imaging using Hadamard-encoded multiple postlabeling delays for the detection of reduced CBF in such patients.
\end{abstract}

MATERIALS AND METHODS: Thirty-seven patients underwent brain perfusion SPECT and pseudocontinuous arterial spin-labeling MR imaging using standard postlabeling delay $(1525 \mathrm{~ms})$ and Hadamard-encoded multiple postlabeling delays. For Hadamard-encoded multiple postlabeling delays, based on data obtained from the 7 sub-boluses with combinations of different labeling durations and postlabeling delays, CBF corrected by the arterial transit time was calculated on a voxel-by-voxel basis. Using a 3D stereotaxic template, we automatically placed ROIs in the ipsilateral cerebellar hemisphere and 5 MCA territories in the symptomatic cerebral hemisphere; then, the ratio of the MCA to cerebellar ROI was calculated.

RESULTS: The area under the receiver operating characteristic curve for detecting reduced SPECT-CBF ratios $(<0.686)$ was significantly greater for the Hadamard-encoded multiple postlabeling delays-CBF ratios (0.885) than for the standard postlabeling delay-CBF ratios (0.786) $(P=.001)$. The sensitivity and negative predictive value for the Hadamard-encoded multiple postlabeling delays-CBF ratios were $100 \%$ (95\% confidence interval, $100 \%-100 \%)$ and significantly higher than the sensitivity $(95 \% \mathrm{Cl}, 44 \%-80 \%)$ and negative predictive value (95\% Cl, 88\%-97\%) for the standard postlabeling delay-CBF ratio, respectively.

CONCLUSIONS: ASL MR imaging using Hadamard-encoded multiple postlabeling delays may be applicable as a screening tool because it can detect reduced CBF on brain perfusion SPECT with 100\% sensitivity and a 100\% negative predictive value in adult patients with ischemic Moyamoya disease.

ABBREVIATIONS: ASL = arterial spin-labeling; ATT = arterial transit time; hASL = Hadamard-encoded multiple PLD ASL; MMD = Moyamoya disease; PLD = postlabeling delay; $S A S L=$ standard-encoded PLD ASL

M oyamoya disease (MMD) is a chronic, occlusive cerebrovascular disease of unknown etiology that is characterized by bilateral steno-occlusive changes in the terminal portion of the ICA and an abnormal vascular network at the base of the brain. ${ }^{1,2}$ The typical treatment for patients with ischemia symptoms is

Received December 28, 2020; accepted after revision March 11, 2021.

From the Department of Neurosurgery (K.S., T.C., S.F., M.K., K. Yoshida, Y. Kubo, K.O.), Department of Radiology (M. Suzuki, K. Yoshioka), and Division of Ultrahigh Field MRI, Institute for Biomedical Sciences (T.M., M. Sasaki), Iwate Medical University School of Medicine, Yahaba-cho, Japan.

This study was funded by a Grant-in-Aid for Scientific Research from the Japan Society for the Promotion of Science (JP18K09002).

Please address correspondence to Kuniaki Ogasawara, MD, Department of Neurosurgery, Iwate Medical University, Idaidoori 2-1-1, Yahaba-cho 020-8505, Japan; e-mail: kuogasa@iwate-med.ac.jp

- Indicates open access to non-subscribers at www.ajnr.org

Indicates article with online supplemental data.

http://dx.doi.org/10.3174/ajnr.A7167 revascularization surgery, which can involve anastomosis of the superficial temporal artery and MCA. ${ }^{3}$ Regardless of the severity of cerebral ischemia, this procedure is universally recommended for pediatric patients with MMD with ischemic symptoms because in such cases, the brain is still developing. ${ }^{3-5}$ By contrast, revascularization surgery is selectively recommended for adult patients presenting with ischemic symptoms. ${ }^{3,6}$ However, no clear guidelines are available for this selection. Two recent studies used PET and brain perfusion SPECT to measure the oxygen extraction fraction and CBF, respectively, and demonstrated that among adult patients (older than 30 but younger than 60 years of age) receiving medication alone for symptomatic ischemic MMD without hemodynamic compromise, such as misery perfusion or reduced $\mathrm{CBF}$, the incidence of recurrent ischemic events was very low (approximately $1 \%$ per year). ${ }^{7,8}$ Moreover, the recurrent ischemic episodes were transient ischemic attacks only, and no changes were seen in scores on the modified Rankin Disability 
Scale after the recurrence of ischemic symptoms in such patients. ${ }^{7}$ In addition, neither cerebral hemodynamics nor cognitive function had deteriorated at 2 years after the last event in patients without recurrent ischemic events. ${ }^{7}$ Initial treatment with medication alone may be recommended for adult patients with ischemic MMD without hemodynamic compromise, ${ }^{7}$ and the determination of whether hemodynamic compromise exists in the symptomatic cerebral hemisphere is important for the management of such patients. ${ }^{7,8}$ However, the clinical use of PET or brain perfusion SPECT to detect misery perfusion or reduced $\mathrm{CBF}$ is precluded by its high cost and limited availability in the clinical setting. Other screening modalities for detecting hemodynamic compromise before performing PET or brain perfusion SPECT would therefore be useful.

Arterial spin-labeling (ASL) MR imaging is a noninvasive technique that can quantify $\mathrm{CBF}^{9-11} \mathrm{~A}$ number of studies involving patients with MMD conducted using ASL MR imaging with a single postlabeling delay (PLD) have simply compared CBF measured using ASL MR imaging with brain perfusion parameters measured using other modalities such as PET, SPECT, and dynamic susceptibility contrast perfusion MR imaging. ${ }^{12-14}$ However, almost all the cerebral cortical regions in MMD have some degree of perfusion delay, which varies even within individual cerebral cortical regions of a single patient. ${ }^{15}$ Therefore, it is difficult to obtain precise CBF measurements using the standard ASL method with a single PLD in MMD. ${ }^{15}$ By contrast, theoretically, ASL measurements covering a wide range of PLD times can be interpreted to estimate CBF with practical spatial and temporal resolutions in the clinical setting. ${ }^{16,17}$ Actually, multi-PLD ASL MR imaging with corrections using the arterial transit time (ATT) significantly improved CBF quantification compared with single-PLD ASL MR imaging. ${ }^{18,19}$ However, the accuracy of multi-PLD ASL MR imaging for detecting hemodynamic compromise has not been reported. Hadamard-encoding techniques have recently been applied to the ASL methods to calculate the ASL signals as multiple PLDs by dividing the ASL signal into sub-boluses of different PLDs. ${ }^{16}$ Several investigations have demonstrated that Hadamard-encoded labeling strategies for healthy human subjects provide robust CBF measurements in a time-efficient manner with shorter scan times $\left(<10\right.$ minutes). ${ }^{20}$ This benefit may be suitable for the detection of reduced CBF in patients with MMD.

Given this background, the aims of this study were to determine the diagnostic accuracy of ASL MR imaging using Hadamardencoded multiple PLDs for the detection of reduced CBF, a key determinant for revascularization surgery, in adult patients with ischemic MMD, and to propose practical clinical algorithms using ASL MR imaging and subsequent management in such patients.

\section{MATERIALS AND METHODS}

The protocol of the present prospective observational study was reviewed and approved by our institutional ethics committee. Written, informed consent was obtained from all participants or their next of kin before the study began.

\section{Patients}

Patients who were diagnosed as having MMD according to the diagnostic criteria of the Research Committee on Spontaneous
Occlusion of the Circle of Willis of the Ministry of Health, Labor, and Welfare of Japan ${ }^{1}$ were prospectively selected for participation in this study if they satisfied the following clinical inclusion criteria: older than 30 but younger than 60 years of age, modified Rankin disability scale score of 0 or 1 , presence of episodes of ischemic symptoms in the unilateral carotid territory occurring $\leq 3$ months before presentation to our department, and absence of infarction in the bilateral cerebral cortices on MR imaging.

\section{Brain Perfusion SPECT}

A brain perfusion SPECT study was performed using $\mathrm{N}$-isopropyl-p- $\left[{ }^{123} \mathrm{I}\right]$ iodoamphetamine $\left({ }^{123} \mathrm{I}\right.$-IMP) and a triple-head gamma camera (GCA-9300R; Toshiba Medical Systems) between 3 weeks and 3 months after the last ischemic event. The spatial resolution of the gamma camera with a high-resolution fan-beam collimator was $8.5-\mathrm{mm}$ full width at half maximum. The SPECT acquisition protocol consisted of a matrix size of $128 \times 128$ and a 30 -minute continuous acquisition (5 minutes/rotation) over $360^{\circ}$ in $4^{\circ}$ steps. Postacquisition, the data were corrected for scatter using the triple energy window method and then reconstructed by filtered back-projection. A Butterworth preprocessing filter was applied with a cutoff frequency of 0.08 cycles per pixel (1 pixel $=1.72 \mathrm{~mm}$ ). For attenuation correction, the iterative Chang method was used, ${ }^{21}$ and the attenuation map was generated by extracting the skin contour and assuming the inner region side as a uniform attenuation body. The attenuation coefficient was $0.146 \mathrm{~cm}^{-1}$.

After delivering a 1-minute intravenous infusions of $222 \mathrm{MBq}$ of ${ }^{123} \mathrm{I}$-IMP (5-mL volume) and physiologic saline at a constant rate of $5 \mathrm{~mL} / \mathrm{min}$, data were acquired at a midscan time of 30 minutes after the administration of ${ }^{123}$ I-IMP with a scan duration of 20 minutes. ${ }^{22}$

\section{ASL MRI}

ASL MR imaging studies were performed using a 3T MR imaging scanner (Discovery MR750; GE Healthcare) with an 8-channel receive head coil and body coil for transmission within 3 days after the brain perfusion SPECT study. Each patient underwent 2 kinds of ASL MR imaging with a 3D pseudocontinuous labeling sequence. At first, the standard ASL (sASL) was performed using the following parameters: labeling duration, $1450 \mathrm{~ms}$; PLD, $1525 \mathrm{~ms}$; TR, $4802 \mathrm{~ms}$; TE, $10.5 \mathrm{~ms}$; section thickness, $4.0 \mathrm{~mm}$; number of slices, 32; sampling points, 8 arms $\times 512$; FOV, 24 $\times 24 \mathrm{~cm}$; matrix size, $128 \times 128$; and voxel resolution, $1.9 \times 1.9$ $\times 4.0 \mathrm{~mm}$. The total scan time was 3 minutes 13 seconds. Next, for ASL MR imaging using Hadamard-encoded multiple PLDs (hASL), we performed ASL consisting of 7 sub-boluses ${ }^{17}$ with the following combinations of labeling duration and PLDs: labeling duration (ms), PLD (ms) = 286, 700; 330, 998; 370, 1327; 432, 1697; 534, 2128; 732, 2661; and 1308, 3392, with TR, $6141 \mathrm{ms;}$ TE, $24.3 \mathrm{~ms}$; section thickness, $4.0 \mathrm{~mm}$; number of slices, 32; sampling points, 4 arms $\times 512$; FOV, $24 \times 24 \mathrm{~cm}$; matrix size, $128 \times 128$; and voxel resolution, $1.9 \times 1.9 \times 4.0 \mathrm{~mm}$. The total scan time was 7 minutes 35 seconds. For each scan, vessel suppression using a velocity-selective saturation sequence was applied to reduce artifactual bright signals by dephasing intravascular labeled spins. ${ }^{20}$ On the basis of data obtained from the 7 sub-boluses, CBF 

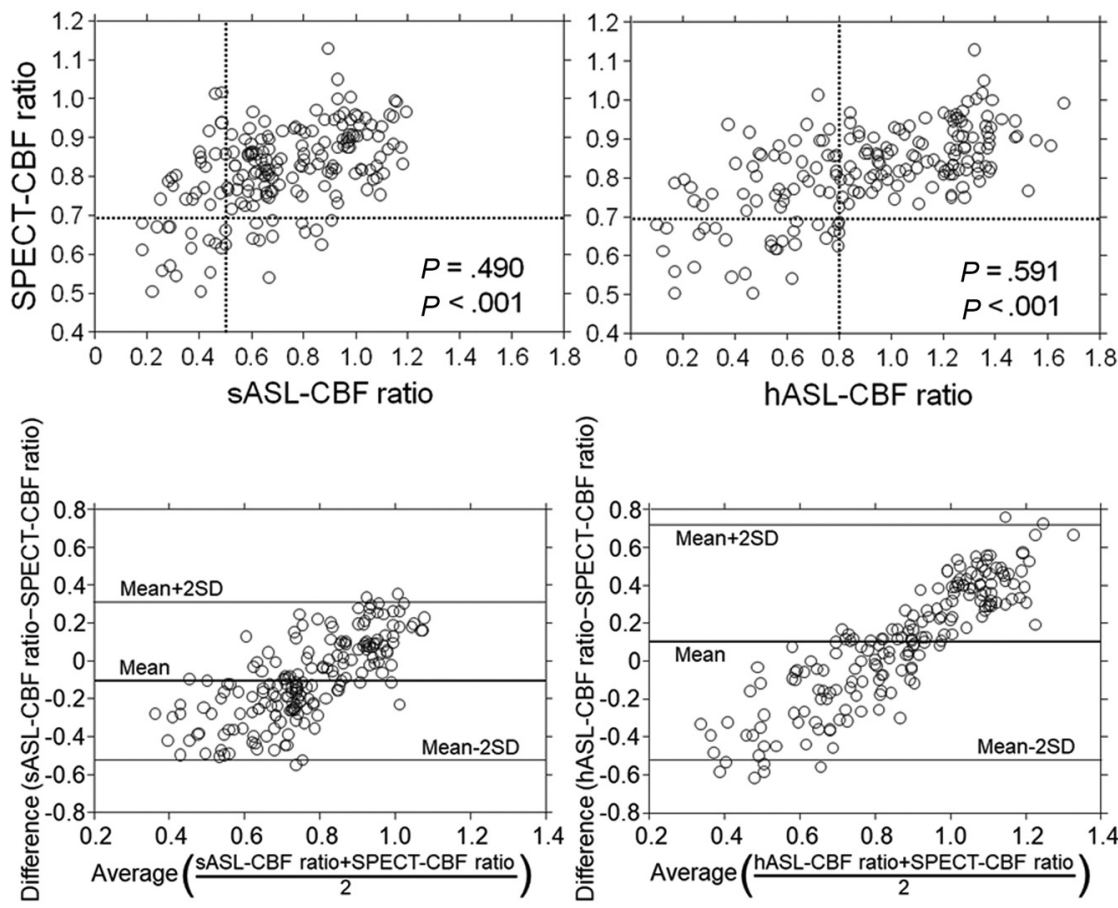

FIG 1. Comparisons (upper graphs) and Bland-Altman plots (lower graphs) of SPECT-CBF and sASL-CBF ratios and sASL-CBF (left graphs) or hASL-CBF ratios (right graphs) for each MCA ROI in each patient. In the upper graphs, each horizontal line denotes the cutoff point for indicating a reduced SPECT-CBF ratio (0.686) and each vertical line denotes the cutoff point lying closest to the upper left corner of the receiver operating characteristic curve for detecting a reduced SPECT-CBF ratio (0.502 for SASL-CBF, 0.801 for hASL-CBF).

corrected by the ATT was automatically calculated on a voxel-byvoxel basis.

\section{Data Analysis and Definition of Reduced CBF}

All SPECT and ASL MR imaging data were transformed into the standard brain size and shape by linear and nonlinear transformation using statistical parametric mapping (SPM2; http:// www.fil.ion.ucl.ac.uk/spm/software/spm12) for anatomic standardization. ${ }^{23}$ Therefore, the brain images from all participants had the same anatomic format. A total of 318 constant ROIs were automatically placed in both the cerebral and cerebellar hemispheres using a 3D stereotaxic ROI template with SPM2. ${ }^{24}$ ROIs were grouped into 10 segments-callosomarginal, pericallosal, precentral, central, parietal, angular, temporal, posterior, hippocampal, and cerebellar-in each hemisphere according to the arterial supply. Of these 10 segments, 5-precentral, central, parietal, angular, and temporal-perfused by the MCA and cerebellar ROI were selected for analyses. ${ }^{22}$

The mean values of the radioactive counts on brain perfusion SPECT images and CBF and ATT on ASL MR images were measured in the 5 MCA ROIs in both cerebral hemispheres and both cerebellar ROIs. For each patient, the mean value in each MCA ROI in the symptomatic cerebral hemisphere relative to that in the ipsilateral cerebellar ROI on brain perfusion SPECT and ASL MR imaging was calculated and defined as the SPECT-CBF, ASL-CBF, and ASL-ATT ratios, respectively.
For each MCA ROI of each patient, the ROI was defined as exhibiting reduced $\mathrm{CBF}$ when the SPECT-CBF ratio was $<0.686 .{ }^{22}$

\section{Statistical Analysis}

Data are expressed as the mean (SD). Correlations between 2 variables were determined using the Spearman rank correlation coefficient. Differences between 2 correlation coefficients were compared using the Meng-RosenthalRubin method. Bland-Altman analysis was performed to confirm the concordance between each $\mathrm{CBF}$ ratio. Taking the correlation between different measures in the same patient into account, we evaluated the relationship between SPECT-CBF and ASL-CBF ratios using generalized linear mixed effects models with a subject random intercept. These 2 parameters were assumed to be a normal variable with an identity link. Receiver operating characteristic curves were used to assess the accuracy of the sASL-CBF and hASL-CBF ratios for detecting reduced $\mathrm{CBF}$. Reduced $\mathrm{CBF}$ as a criterion standard was defined as a SPECT-CBF ratio of $<0.686 .{ }^{22}$ Pair-wise comparisons of the area under the receiver operating characteristic curves for the sASL-CBF and hASL-CBF ratios were then performed. For all statistical analyses, significance was set at $P<.05$. The exact 95\% CIs for sensitivity, specificity, and positive and negative predictive values were computed with binomial distributions. Differences in the sensitivity, specificity, and positive and negative predictive values among the sASL-CBF and hASLCBF ratios were analyzed using 95\% CIs.

\section{RESULTS}

During the 24-month study period, a total of 40 patients met the inclusion criteria, among whom, 3 declined to participate. The remaining 37 patients (13 men, 24 women; mean age, 45 [SD, 6] years; age range, 31-57 years) underwent brain perfusion SPECT and ASL MR imaging, leading to $185(5 \times 37)$ MCA ROIs in the symptomatic cerebral hemispheres being included in the final analyses. Among these 37 patients, 29 experienced the onset of transient ischemic attacks alone; 3 , a minor stroke alone; and 5 , onset of a minor stroke after a transient ischemic attack. In total, 11,16 , and 10 patients were classified into MRA stages 2,3 , and 4 , respectively. ${ }^{1}$

A significant positive correlation was observed between SPECT-CBF and sASL-CBF $(\rho=0.490 ; P<.001)$ or hASL-CBF ratios ( $\rho=0.591 ; P<.001)$ in the 185 MCA ROIs (Fig 1$)$. The latter coefficient was significantly greater than the former $(P=.002)$. A significant positive correlation was also observed between SPECT-CBF ratios and the quantitative values of 


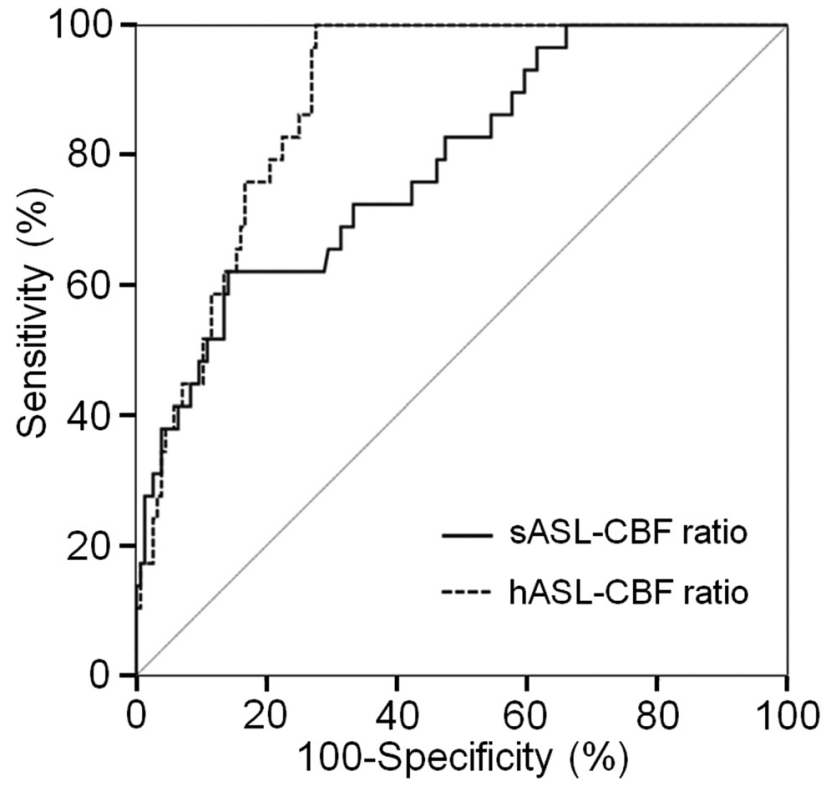

FIG 2. Receiver operating characteristic curves used to compare the accuracy of SASL-CBF and hASL-CBF ratios for detecting a reduced SPECT-CBF ratio. Pair-wise comparison analysis shows a significantly greater area under the receiver operating characteristic curve for the hASL-CBF (dotted line) than for the SASL-CBF ratio (solid line).

SASL-CBF $(\rho=0.462 ; P<.001)$ or hASL-CBF $(\rho=0.534$; $P<.001$ ) in the 185 MCA ROIs (Online Supplemental Data). Although the former coefficient did not differ from that between SPECT-CBF and SASL-CBF ratios, the latter coefficient was significantly lower than that between SPECT-CBF and hASL-CBF ratios $(P=.036)$.

No constant bias was detected for the SPECT-CBF and sASLCBF (95\% CI of difference, $-0.530-0.319$ ) or hASL-CBF ratios (95\% CI of difference, $-0.510-0.711$ ) by Bland-Altman analysis (Fig 1). However, a proportional bias was identified for SPECTCBF and sASL-CBF (slope-of-regression equation, $0.969 ; P<.001$ ) or hASL-CBF ratios (slope-of-regression equation, $1.242 ; P<.001$ ) (Fig 1). Whereas SASL-CBF ratios showed a propensity toward underestimation at lower CBF ratios, hASL-CBF ratios showed a propensity toward underestimation and overestimation at lower and higher $\mathrm{CBF}$ ratios, respectively. Generalized linear mixed effects models with a subject random intercept revealed a significant relationship between SPECT-CBF and sASL-CBF $(P<.001)$ or hASL-CBF ratios $(P<.001)$.

Of the 185 ROIs analyzed, 29 (16\%) were determined to exhibit reduced $\mathrm{CBF}$ on brain perfusion SPECT. The areas under the curve for sASL-CBF and hASL-CBF ratios for detecting reduced CBF on brain perfusion SPECT were 0.786 (95\% CI, 0.720-0.843) and 0.885 (95\% CI, 0.830-0.927), respectively; the latter was significantly greater than the former (difference between areas, 0.099; $P=.001$ ) (Fig 2).

The sensitivity and negative predictive value for the hASL$\mathrm{CBF}$ ratio, with the cutoff point lying closest to the upper left corner of the receiver operating characteristic curve for the detection of MCA ROIs with reduced CBF on brain perfusion SPECT, were $100 \%$ (cutoff point, 0.801 ) and significantly higher than those for the sASL-CBF ratio (cutoff point, 0.502) (Online
Supplemental Data). The specificity and positive predictive value did not differ between the 2 ASL-CBF ratios.

When the difference between the hASL-CBF and SPECT-CBF ratios (hASL-CBF ratio-SPECT-CBF ratio) was compared with the hASL-ATT ratio for each MCA ROI (Online Supplemental Data), the differences in most MCA ROIs exhibiting nonreduced hASL-CBF ratios $(\geq 0.801)$ and nonreduced SPECT-CBF ratios $(\geq 0.686)$ were $>0$, regardless of the hASL-ATT ratios. By contrast, the differences for most MCA ROIs with reduced hASLCBF ratios $(<0.801)$ were $<0$ regardless of the ASL-ATT ratios. In particular, no MCA ROIs with reduced hASL-CBF ratios exhibited reduced SPECT-CBF ratios when they had an hASLATT ratio of $>1.3$.

Of the 37 patients studied, 1, 3, 6, and 4 patients had 4, 3, 2, and 1 MCA ROIs with reduced CBF on brain perfusion SPECT, respectively. Therefore, 14 patients $(38 \%)$ were determined to have MCA ROIs with reduced CBF. In the 10 patients with $\geq 2$ MCA ROIs with reduced CBF, the ROIs were adjacent to one another. The sensitivity and negative predictive value for the hASL-CBF ratio for the detection of patients with MCA ROIs exhibiting reduced $\mathrm{CBF}$ on brain perfusion SPECT based on the determination for each MCA ROI were $100 \%$ and significantly higher than those for the sASL-CBF ratio (Online Supplemental Data). The specificity and positive predictive value did not differ between these 2 ASL-CBF ratios.

Figures 3 and 4 show representative MRA, brain perfusion SPECT, and ASL MR images in a patient with MCA ROIs exhibiting reduced $\mathrm{CBF}$ and in a patient without any MCA ROIs exhibiting reduced $\mathrm{CBF}$, respectively.

\section{DISCUSSION}

The results of this study demonstrate that ASL MR imaging using Hadamard-encoded multiple PLDs can detect reduced CBF on brain perfusion SPECT with $100 \%$ sensitivity and negative predictive value in adult patients with ischemic MMD. This diagnostic accuracy may be applicable as a screening test before further examination of cerebral hemodynamics.

In this study, CBF in each MCA ROI in the symptomatic cerebral hemisphere was normalized using $\mathrm{CBF}$ in the ipsilateral cerebellar hemisphere. This cerebellar normalization has been commonly applied to brain perfusion SPECT because of its simplicity, objectivity, and reproducibility. ${ }^{22,25,26}$ By contrast, the accuracy of the cerebellar normalization for ASL-CBF in patients with MMD remains controversial. One previous study demonstrated improvement of the correlation between sASL-CBF and PET-CBF by cerebellar normalization, ${ }^{15}$ whereas another found overestimation of sASL-CBF by cerebellar normalization. ${ }^{12}$ Perfusion delay may differ between the cerebral and cerebellar cortexes in healthy subjects, and this difference in perfusion delay may vary in patients with MMD. Our data showed a better correlation between SPECT$\mathrm{CBF}$ ratios and hASL-CBF ratios compared with that between SPECT-CBF ratios and SASL-CBF ratios or between SPECT-CBF ratios and quantitative hASL-CBF values. These findings suggest that correcting brain perfusion delay, including the cerebellum using Hadamard-encoded multiple PLDs, may increase the accuracy of cerebellar normalization for ASL-CBF in MMD. However, this correction also underestimated and overestimated ASL-CBF 

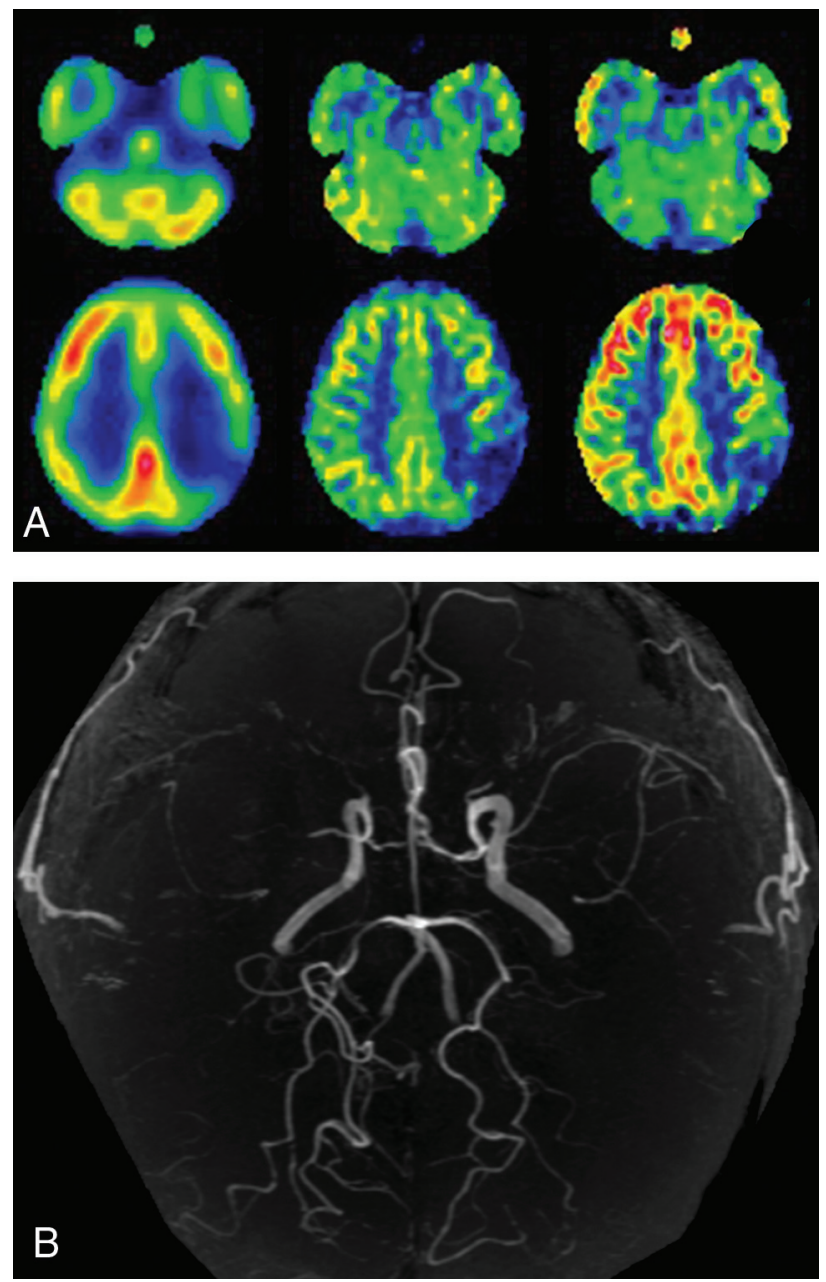

FIG 3. MRA ( $B$ ), brain perfusion SPECT images ( $A$, left), and SASL ( $A$, middle) and hASL (A, right) MR images of a 43-year-old male patient with transient ischemic attacks complicated by right-sided hemiparesis. MRA shows stage 3 Moyamoya disease. On all 3 SPECT and ASL images, the CBF in the left parietal region is severely reduced compared with that in the left cerebellar hemisphere.

ratios at lower and higher CBFs, respectively. We used the vesselsuppression technique for hASL MR imaging to reduce artifactual bright signals by dephasing intravascular labeled spins. ${ }^{27}$ However, this technique theoretically prolongs the effective TE, resulting in a reduction in the SNR. ${ }^{27}$ Such SNR reductions may be related to the underestimation of hASL-CBF ratios at lower CBFs. Actually, investigators do not necessarily recommend the use of vessel suppression for clinical applications. ${ }^{27}$ By contrast, the overestimation of hASL-CBF ratios at higher CBFs may be due to the overcorrection of cerebral perfusion delay using Hadamard-encoded multiple PLDs in cerebral regions with slightly or not prolonged PLDs; these should be considered limitations of hASL MR imaging.

A previous study using PET demonstrated that among adult patients receiving medication alone for symptomatic ischemic MMD without an increased oxygen extraction fraction in all MCA ROIs, the incidence of recurrent ischemic events was very low. ${ }^{7}$ Another study showed that SPECT-CBF ratios obtained in the same fashion as in the present study detect increased oxygen
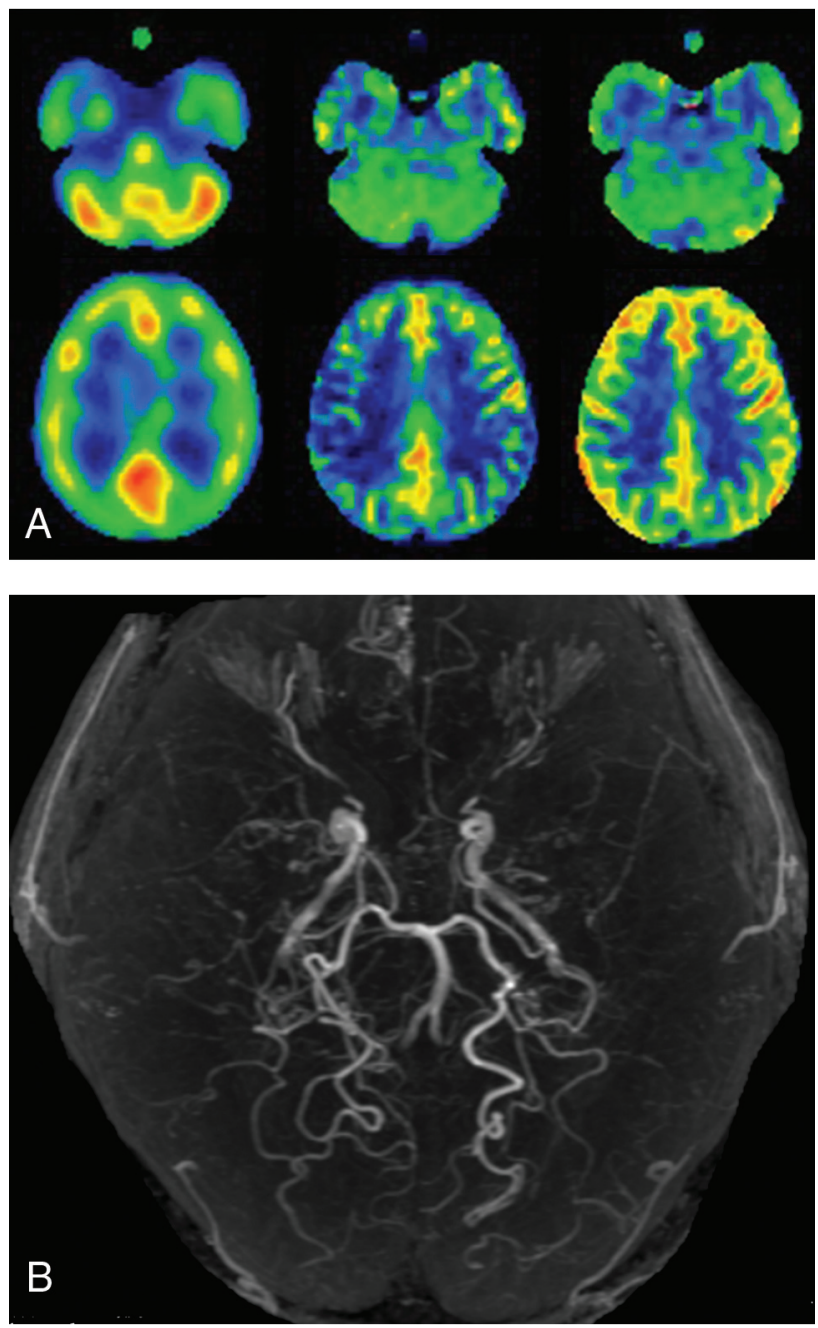

FIG 4. MRA ( $B)$, brain perfusion SPECT images ( $A$, left), and sALS ( $A$, middle) and hASL (A, right) MR images of a 34-year-old female patient with transient ischemic attacks complicated by left-sided hemiparesis. MRA shows stage 3 Moyamoya disease. On perfusion SPECT images, the CBF in the right MCA territory is slightly reduced compared with that in the right cerebellar hemisphere. The CBF on SASL MR images is severely reduced in the right central, parietal, and angular regions, whereas the CBF on hASL MR imaging is not reduced relative to the right cerebellar hemisphere.

extraction fraction with $100 \%$ sensitivity and a $100 \%$ negative predictive value in adult patients with ischemic MMD (older than 30 but younger than 60 years of age), and the optimal cutoff point for the SPECT-CBF ratio for detection of increased oxygen extraction fraction is $0.686 .{ }^{22}$ The present study, therefore, defined a SPECT-CBF ratio below this cutoff point as reduced $\mathrm{CBF}$. As a result, the sensitivity and negative predictive value of hASL for the detection of reduced CBF on brain perfusion SPECT were $100 \%$. This diagnostic accuracy may be applicable to a screening test before further examination of cerebral hemodynamics. By contrast, the positive predictive value of hASL was low $(<50 \%)$ and comparable with that of sASL. In particular, reduced CBF on hASL was not corrected when the hASL-ATT ratio was $>1.3$. This finding suggests that hASL does not

AJNR Am J Neuroradiol 42:1403-09 Aug 2021 www.ajnr.org

1407 
sufficiently correct the longer perfusion delays in the cerebral cortex in patients with MMD, also a limitation of hASL MR imaging.

On the basis of the present data and previous findings, ${ }^{7,22}$ we propose practical clinical algorithms for the detection of hemodynamic compromise using ASL MR imaging with Hadamardencoded multiple PLDs and subsequent management in adult patients with ischemic MMD. Patients who undergo ASL MR imaging using Hadamard-encoded multiple PLDs are determined to not have hemodynamic compromise and should, therefore, receive medication alone, including an antiplatelet drug, if the ASL-CBF ratios in all MCA ROIs in the symptomatic hemisphere are not low $(\geq 0.801)$. If the ASL-CBF ratio in any MCA ROI is low $(<0.801)$, the patient may possibly have hemodynamic compromise and, therefore, requires further examinations such as brain perfusion SPECT and/or $\left({ }^{15} \mathrm{O}\right)$ gas PET to detect hemodynamic compromise more accurately. If hemodynamic compromise is detected on SPECT or PET, revascularization surgery may be recommended. The introduction of ASL MR imaging using Hadamard-encoded multiple PLDs for screening may reduce the number of patients who undergo PET or brain perfusion SPECT by about two-thirds.

A prospective study ${ }^{7}$ reported that during the recurrence of ischemic events in adult patients with MMD, the affected cerebral hemisphere exhibited new misery perfusion. By contrast, cerebral hemodynamics had not deteriorated 2 years after the last event in patients without recurrent ischemic events. Those results suggest that the development of misery perfusion may be a strong predictor of recurrent ischemic events in adult patients with ischemiconset MMD. ${ }^{7}$ The detection of reduced CBF using screening ASL MR imaging with Hadamard-encoded multiple PLDs for such patients may be also recommended for the prediction of recurrent ischemic symptoms during follow-up.

In addition to the limitations listed above, we did not assess stress perfusion such as acetazolamide challenge. A previous study demonstrated that whereas $\mathrm{CBF}$ assessed using brain perfusion SPECT detects misery perfusion with high sensitivity and a high negative predictive value in adult patients with ischemic MMD, the accuracy of cerebrovascular reactivity to acetazolamide is significantly lower than that of $\mathrm{CBF}^{22}$ Furthermore, cerebrovascular reactivity to acetazolamide in addition to $\mathrm{CBF}$ did not increase the accuracy for detecting misery perfusion. ${ }^{22}$ These findings suggest that hemodynamic compromise can be sufficiently detected by $\mathrm{CBF}$ measurement alone in such patients. ${ }^{22}$

\section{CONCLUSIONS}

ASL MR imaging using Hadamard-encoded multiple PLDs can detect reduced CBF on brain perfusion SPECT with 100\% sensitivity and $100 \%$ negative predictive value in adult patients with ischemic MMD; therefore, it may be applicable as a screening tool before further examination of cerebral hemodynamics.

Disclosures: Makoto Sasaki-UNRELATED: Grants/Grants Pending: Japan Society for the Promotion of Science-Kakenhi, Hitachi, GE Healthcare*; Payment for Lectures Including Service on Speakers Bureaus: Hitachi, GE Healthcare. *Money paid to the institution.

\section{REFERENCES}

1. Research Committee on the Pathology and Treatment of Spontaneous Occlusion of the Circle of Willis; Health Labour Sciences Research Grant for Research on Measures for Intractable Diseases. Guidelines for diagnosis and treatment of moyamoya disease (spontaneous occlusion of the circle of Willis. Neurol Med Chir (Tokyo) 2012;52:24566 CrossRef Medline

2. Suzuki J, Takaku A. Cerebrovascular "moyamoya” disease: disease showing abnormal net-like vessels in base of brain. Arch Neurol 1969;20:288-99 CrossRef Medline

3. Fujimura M, Tominaga T. Current status of revascularization surgery for moyamoya disease: special consideration for its 'internal carotid-external carotid (IC-EC) conversion' as the physiological reorganization system. Tohoku J Exp Med 2015;236:45-53 CrossRef Medline

4. Fujimura M, Shimizu H, Mugikura S, et al. Delayed intracerebral hemorrhage after superficial temporal artery-middle cerebral artery anastomosis in a patient with moyamoya disease: possible involvement of cerebral hyperperfusion and increased vascular permeability. Surg Neurol 2009;71:223-27 CrossRef Medline

5. Fushimi Y, Okada T, Takagi Y, et al. Voxel based analysis of surgical revascularization for moyamoya disease: pre- and postoperative SPECT studies. PLoS One 2016;11:e0148925 CrossRef Medline

6. Guzman R, Lee M, Achrol A, et al. Clinical outcome after 450 revascularization procedures for moyamoya disease. J Neurosurg 2009;111:927-35 CrossRef Medline

7. Miyoshi K, Chida K, Kobayashi M, et al. Two-year clinical, cerebral hemodynamic and cognitive outcomes of adult patients undergoing medication alone for symptomatically ischemic moyamoya disease without cerebral misery perfusion: a prospective cohort study. Neurosurgery 2018;84:1233-41 CrossRef Medline

8. Nam KW, Cho WS, Kwon HM, et al. Ivy sign predicts ischemic stroke recurrence in adult moyamoya patients without revascularization surgery. Cerebrovasc Dis 2019;47:223-30 CrossRef Medline

9. Williams DS, Detre JA, Leigh JS, et al. Magnetic resonance imaging of perfusion using spin inversion of arterial water. Proc Natl Acad Sci USA 1992;89:212-16 CrossRef Medline

10. Alsop DC, Detre JA. Reduced transit-time sensitivity in noninvasive magnetic resonance imaging of human cerebral blood flow. $J$ Cereb Blood Flow Metab 1996;16:1236-49 CrossRef Medline

11. Detre JA, Samuels OB, Alsop DC, et al. Noninvasive magnetic resonance imaging evaluation of cerebral blood flow with acetazolamide challenge in patients with cerebrovascular stenosis. J Magn Reson Imaging 1999;10:870-75 CrossRef Medline

12. Goetti R, Warnock G, Kuhn FP, et al. Quantitative cerebral perfusion imaging in children and young adults with moyamoya disease: comparison of arterial spin-labeling-MRI and $\mathrm{H}(2)[(15) \mathrm{O}]-$ PET. AJNR Am J Neuroradiol 2014;35:1022-28 CrossRef Medline

13. Yun TJ, Sohn $\mathrm{CH}$, Han $\mathrm{MH}$, et al. Effect of delayed transit time on arterial spin labeling: correlation with dynamic susceptibility contrast perfusion magnetic resonance in moyamoya disease. Invest Radiol 2013;48:795-802 CrossRef Medline

14. Noguchi $T$, Kawashima $M$, Irie $H$, et al. Arterial spin-labeling MR imaging in moyamoya disease compared with SPECT imaging. Eur J Radiol 2011;80:e557-62 CrossRef Medline

15. Hara S, Tanaka Y, Ueda Y, et al. Noninvasive evaluation of CBF and perfusion delay of moyamoya disease using arterial spin-labeling MRI with multiple postlabeling delays: comparison with 15O-gas PET and DSC-MRI. AJNR Am J Neuroradiol 2017;38:696-702 CrossRef Medline

16. Gunther M. Highly efficient accelerated acquisition of perfusion inflow series by cycled arterial spin labeling. In: Proceedings of the 15th Annual Meeting of International Society of Magnetic Resonance in Medicine and the European Society for Magnetic Resonance in Medicine and Biology, Berlin, Germany; May 19-25, 2007

17. Wells JA, Lythgoe MF, Gadian DG, et al. In vivo Hadamard-encoded continuous arterial spin-labeling (H-CASL). Magn Reson Med 2010;63:1111-18 CrossRef Medline 
18. Fan AP, Khalighi MM, Guo J, et al. Identifying hypoperfusion in moyamoya disease with arterial spin labeling and an [15O]-water positron emission tomography/magnetic resonance imaging normative database. Stroke 2019;50:373-80 CrossRef Medline

19. Wang R, Yu S, Alger JR, et al. Multi-delay arterial spin-labeling perfusion MRI in moyamoya disease: comparison with CT perfusion imaging. Eur Radiol 2014;24:1135-44 CrossRef Medline

20. Guo J, Holdsworth SJ, Fan AP, et al. Comparing accuracy and reproducibility of sequential and Hadamard-encoded multidelay pseudocontinuous arterial spin-labeling for measuring cerebral blood flow and arterial transit time in healthy subjects: a simulation and in vivo study. J Magn Reson Imaging 2018;47:1119-32 CrossRef Medline

21. Chang LT. A method for attenuation correction in radionuclide computed tomography. IEEE Trans Nucl Sci 1978;25:638-43 CrossRef

22. Setta K, Kojima D, Shimada Y, et al. Accuracy of brain perfusion single-photon emission computed tomography for detecting misery perfusion in adult patients with symptomatic ischemic moyamoya disease. Ann Nucl Med 2018;32:611-19 CrossRef Medline
23. Nishimiya M, Matsuda H, Imabayashi E, et al. Comparison of SPM and NEUROSTAT in voxelwise statistical analysis of brain SPECT and MRI at the early stage of Alzheimer's disease. Ann Nucl Med 2008;22:921-27 CrossRef Medline

24. Takeuchi R, Matsuda H, Yoshioka K, et al. Cerebral blood flow SPET in transient global amnesia with automated ROI analysis by 3DSRT. Eur J Nucl Med Mol Imaging 2004;31:578-89 CrossRef Medline

25. Soonawala D, Amin T, Ebmeier KP, et al. Statistical parametric mapping of $(99 \mathrm{~m}) \mathrm{Tc}-\mathrm{HMPAO}$-SPECT images for the diagnosis of Alzheimer's disease: normalizing to cerebellar tracer uptake. Neuroimage 2002;17:1193-1202 CrossRef Medline

26. Ohnishi T, Yano T, Nakano S, et al. Acetazolamide challenge and technetium-99m-ECD versus iodine-123-IMP SPECT in chronic occlusive cerebrovascular disease. J Nuc Med 1997;38:1463-67 Medline

27. Alsop DC, Detre JA, Golay X, et al. Recommended implementation of arterial spin-labeled perfusion MRI for clinical applications: a consensus of the ISMRM Perfusion Study Group and the European Consortium for ASL in dementia. Magn Reson Med 2015;73:102-16 CrossRef Medline 